\title{
Konzepte zur Qualitätssicherung im Management des Lungenkarzinoms
}

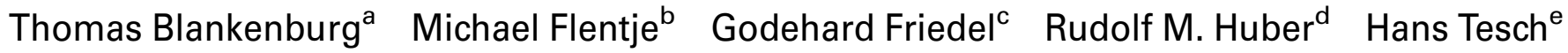 \\ ${ }^{a}$ Klinik für Innere Medizin II; Städtisches Krankenhaus Martha-Maria Halle Dölau, Halle/Saale, \\ ${ }^{b}$ Klinik für Strahlentherapie, Universität Würzburg, \\ ${ }^{c}$ Abteilung für Thoraxchirurgie, Klinik Schillerhöhe, Gerlingen, \\ ${ }^{\mathrm{d}}$ Medizinische Klinik Innenstadt, Universität München, \\ ${ }^{\text {e } G e m e i n s c h a f t s p r a x i s ~ D r s . ~ K l i p p s t e i n, ~ G r u n e w a l d, ~ T e s c h, ~ K n a u f ~ H a ̈ m a t o l o g i e ~ u n d ~ O n k o l o g i e, ~ F r a n k f u r t / M ., ~ D e u t s c h l a n d ~}$
}

Schlüsselwörter

Nicht kleinzelliges Lungenkarzinom · Qualitätssicherung HALLUCA-Studie

Key Words

NSCLC $\cdot$ Quality assurance $\cdot$ HALLUCA study

Den ursprünglich aus der Industrie stammenden Begriff der Qualitätssicherung versucht man seit Ende der 1980er Jahre auch in der Medizin zu implementieren. Denn trotz des zunehmenden Kostendiktats im Gesundheitswesen muss sichergestellt werden, dass jeder Versicherte Zugang zu einer definierten Qualität der Gesundheitsversorgung besitzt. Gefordert sind dabei nicht nur die großen Kliniken, sondern auch die Arztpraxen.

\section{Qualitätssicherung in der Arztpraxis}

Niedergelassene Onkologen, die sich in Schwerpunktpraxen zusammengeschlossen haben, sind für die wohnortnahe Versorgung von Tumorpatienten unverzichtbar. Sie werden repräsentiert durch den Berufsverband der Niedergelassenen Hämatologen und internistischen Onkologen (BNHO), in dem bundesweit über 240 Praxen mit 439 Hämatologen und Onkologen organisiert sind. Mitte 2005 gründete der BNHO das Wissenschaftliche Institut der Hämatologen und Onkologen (WINHO), zu dessen zentralen Aufgaben die Qualitätssicherung, die Entwicklung von Methoden zur Versorgungsforschung sowie die Berechnung von Fallkosten und die Erstellung von GKV-Kostenmodellen (Gesundheitsökonomie) gehören - in der Hoffnung, so die Rahmenbedingungen für die Versorgung von Tumorpatienten zu optimieren. Ende 2005 wurde in Zusammenarbeit mit den Kassenärztlichen Vereinigungen der erste Qualitätssicherungsbericht erstellt.

Die 143 an dem Bericht teilnehmenden Schwerpunktpraxen behandelten im 3. Quartal 2004 über 120000 Tumorpatienten; im Median waren es 250 Patienten pro Arzt und Praxis. Dabei stand das Lungenkarzinom in der Häufigkeit an vierter Stelle hinter dem Mammakarzinom, den malignen Lymphomen und dem kolorektalem Karzinom. In 69 der 143 teilnehmenden Praxen gehörte das Lungenkarzinom zu den fünf häufigsten Tumorentitäten. In diesen 69 Praxen wurden im Erhebungsquartal $2950 \mathrm{~Pa}-$ tienten mit Lungenkarzinom behandelt. Im Mittel behandelte jeder Arzt in einer dieser Praxen 43 Lungenkarzinompatienten pro Quartal (Abb. 1).

\section{Therapiestandards durch Studienteilnahme sichern}

Während des letztjährigen Kongresses der Deutschen Gesellschaft für Hämatologie und Onkologie (DGHO) wurde zudem eine Studienplattform gegründet. Sie soll in einem ersten Schritt klären, inwieweit klinische Studien in Praxen durchführbar bzw. welche Studien für den niedergelassenen Bereich besonders geeignet sind. Im Weiteren sollen dann Studienwerkzeuge, d.h. EDV, Monitoringund Dokumentationsinstrumente, bewertet bzw. verbessert und effiziente Arbeitsgruppen zu den großen Tumorentitäten etabliert werden. Die Studienplattform versteht sich nicht als Konkurrenz zu etablierten Studiengruppen, sondern sucht vielmehr die intensive Kooperation z. B. mit der Arbeitsgemeinschaft Internistische Onkologie (AIO) oder der German Breast Group (GBG). Eine regere Teilnahme an Studien sollte gewährleisten, dass mehr Patienten leitliniengemäß behandelt werden, und zudem generell das Leistungsniveau in Praxen anheben.

\section{KARGER}

Fax +497614520714

E-mail Information@Karger.de

www.karger.com (c) 2006 S. Karger GmbH, Freiburg

Accessible online at: www.karger.com/onk 


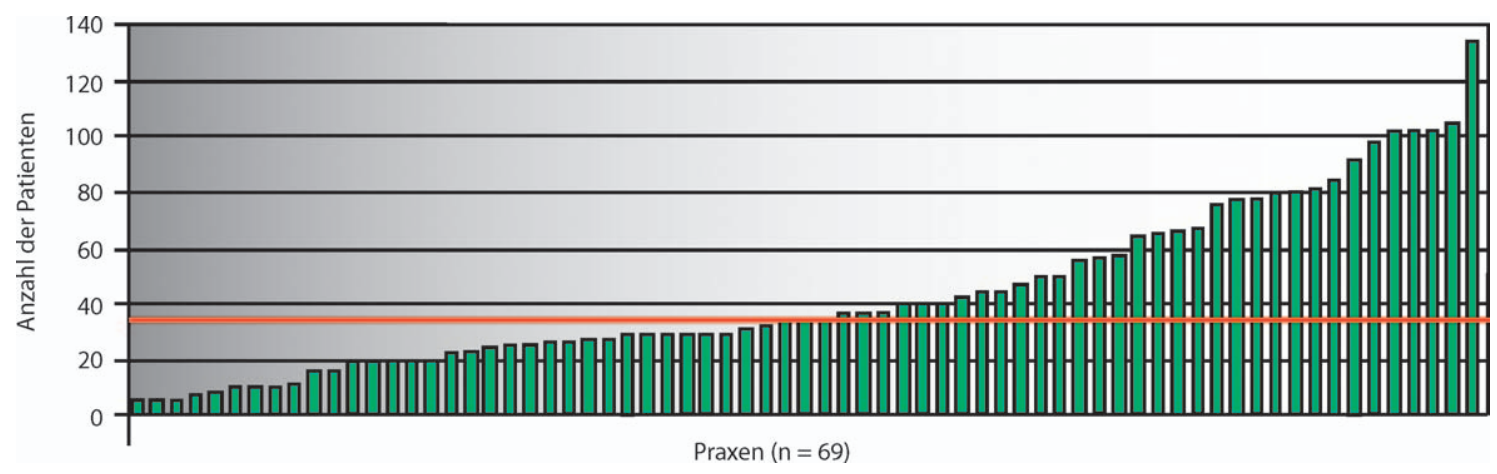

Abb. 1. Häufigkeit von Bronchialkarzinompatienten in onkologischen Schwerpunktpraxen (Tesch).

\section{HALLUCA-Studie zur regionalen Versorgung}

Dass die Evaluierung der Versorgungsqualität beim Lungenkarzinom unter den Bedingungen einer Feldstudie möglich ist, hat die populationsbezogene HALLUCA-Studie im südlichen SachsenAnhalt gezeigt. Diese Studie hat aber auch deutlich gemacht, dass Datendichte und -struktur für eine Qualitätssicherung bislang nicht ausreichen.

An der vom Bundesministerium für Gesundheit geförderten Untersuchung beteiligten sich 3 Universitätskliniken, 16 Krankenhäuser der Grund-, Spezial- und Schwerpunktversorgung, 12 spezialisierte Praxen, 3 Pathologen sowie Hausärzte. Innerhalb von 3,5 Jahren wurden knapp 1700 Patienten erfasst und über mindestens ein Jahr nachbeobachtet.

Anhand hochgerechneter Daten auf Basis des Krebsregisters der DDR waren im Studienzeitraum 2299 Lungenkarzinome erwartet worden. Tatsächlich bekannt wurden 1967 Fälle $(85,6 \%)$, mit Einwilligung auswertbar waren 1696 (73,8\%). In universitären Einrichtungen wurden $8 \%$ der Lungenkarzinome, in Zentren mit Studienerfahrung 61\% diagnostiziert. Die Fehlzahlen bei Diagnostik und Dokumentation lassen sich vermutlich in erster Linie durch die übrigen teilnehmenden Zentren erklären, die ein knappes Drittel (31\%) der in der HALUCCA-Studie registrierten Patienten diagnostizierten. Hierbei handelt es sich um Zentren, die sich nicht an Studien beteiligen und denen es möglicherweise auch an der Kapazität zur vollständigen Dokumentation mangelt. Histologische Angaben nach abgeschlossener Diagnostik fehlten bei rund $8 \%$ der Patienten, insbesondere in den Stadien I und IV. Vollständige Angaben zu Histologie und Tumorstadium wurden in 73,4\% der Fälle geliefert (Tab. 1). Diese Zahl ist allerdings per se wenig aussagekräftig, da Referenzdaten aus der Versorgungsforschung fehlen. Der Vergleich von klinischem (cTNM) und pathologischem (pTNM) TNM-Stadium macht jedoch deutlich, dass nur in $43 \%$ der Fälle präoperativ eine klinisch korrekte Diagnose gestellt wurde. In 35\% wurde das Stadium überschätzt, in $22 \%$ unterschätzt (Tab. 2). Diese Zahlen sind im internationalen Vergleich durchaus akzeptabel (Tab. 3) [1]. Allerdings wurde die Qualität der Diagnostik nicht im Gesamtkollektiv, sondern nur an einer kleinen Subgruppe von $172 \mathrm{~Pa}-$ tienten $(10,1 \%)$ analysiert.
Tab. 1. HALLUCA-Studie: Angaben zu Histologie und Tumorstadium

\begin{tabular}{lccc}
\hline Angaben zur Histologie & \multicolumn{3}{c}{ Angaben zum Tumorstadium } \\
\cline { 2 - 4 } & vorhanden & fehlend & gesamt \\
\hline Vorhanden & & & \\
$\quad$ Anzahl & 1245 & 304 & 1549 \\
$\quad \%$ & 73,4 & 17,9 & 91,3 \\
Fehlend & & & \\
$\quad$ Anzahl & 73 & 74 & 147 \\
$\quad \%$ & 4,3 & 4,4 & 8,7 \\
Gesamt & & & \\
Anzahl & 1318 & 378 & 1696 \\
$\%$ & 77,71 & 22,29 & 100 \\
\hline
\end{tabular}

Tab. 2. HALLUCA-Studie: Vergleich Stadieneinteilung nach cTNM vs. pTNM bei den primär operierten Patienten

\begin{tabular}{lrrrrrrl}
\hline \multirow{2}{*}{$\begin{array}{l}\text { Stadium nach } \\
\text { cTNM }\end{array}$} & \multicolumn{6}{l}{ Stadium nach } \\
\cline { 2 - 8 } & Ia & Ib & IIa & IIb & IIIa & IIIb & IV \\
\hline Ia & 17 & 9 & 3 & 3 & 3 & 2 & 1 \\
Ib & 10 & 24 & 0 & 10 & 11 & 1 & 1 \\
IIa & 0 & 0 & 4 & 1 & 1 & 0 & 1 \\
IIb & 3 & 5 & 0 & 10 & 7 & 1 & 0 \\
IIIa & 2 & 9 & 0 & 9 & 19 & 3 & 2 \\
\hline
\end{tabular}

Tab. 3. HALLUCA-Studie: Korrektheit Stadieneinschätzung entsprechend cTNM

\begin{tabular}{lll}
\hline & HALLUCA & Roberts et al., 1999 [1] \\
\hline Korrektes Stadium, \% & 43 & 42 \\
Unterschätztes Stadium, \% & 22,1 & 30 \\
Überschätztes Stadium, \% & 34,9 & 28 \\
\hline
\end{tabular}

\section{Einrichtungsbezogene Vergleiche schwierig}

Ein exemplarischer Vergleich von 9 verschiedenen Einrichtungen weist auf eine beachtliche Variabilität der erfassten diagnostischen Daten hin. Die einrichtungsbezogene Patientenselektion erschwert die Einschätzung der diagnostischen Qualität zudem erheblich. Eine auf den ersten Blick als Dokumentationsdefizit erscheinende unvollständige Datenerfassung muss sich demzufolge - anders als in klinischen Studien - nicht zwangsläufig in einem Betreuungsdefizit niederschlagen. 


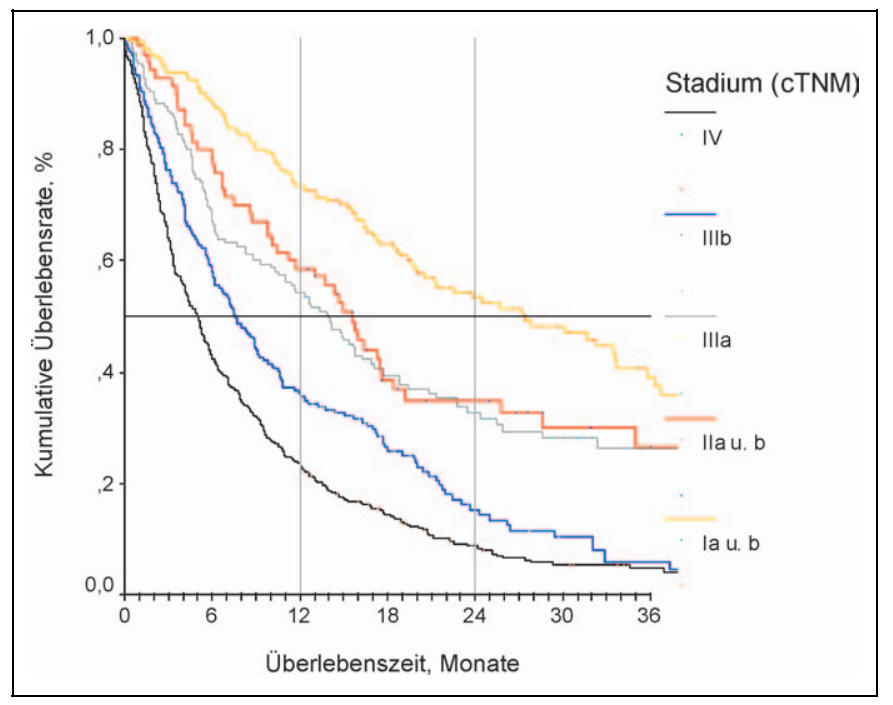

Abb. 2. HALLUCA-Studie: Kaplan-Meier-Überlebenskurven für die einzelnen cTNM-Stadien (Blankenburg).

Tab. 4. HALLUCA vs. Mountain, 1997 [2]: 3-Jahres-Überlebensraten für die einzelnen cTNM-Stadien

\begin{tabular}{lll}
\hline cTNM-Stadium & \multicolumn{2}{l}{ 3-Jahres-Überlebensrate, \% } \\
\cline { 2 - 3 } & HALLUCA & Mountain, 1997 [2] \\
\hline Ia & 40 & 71 \\
Ib & 39 & 46 \\
IIa & 43 & 38 \\
IIb & 24 & 33 \\
IIIa & 26 & 18 \\
IIIb & 6 & 7 \\
IV & 5 & 2 \\
\hline
\end{tabular}

Zur Evaluierung der Behandlungsqualität wurde das therapeutische Vorgehen bei 387 Patienten (22,8\%) mit operablen Lungenkarzinomen (Stadium I-IIIA) analysiert. Der Anteil operierter Patienten war in der HALLUCA-Studie mit nur 53\% niedrig. Bei den nicht operierten Patienten war der Therapieentscheid in mehr als der Hälfte der Fälle diskutabel. Eine derart detaillierte Datenanalyse wie in der Studie ist als Routinemaßnahme im klinischen Alltag aufgrund des hohen personellen Aufwands bedauerlicherweise nicht realisierbar, obwohl sie letztlich zu einer verbesserten Therapiequalität führen sollte.

Auch die Behandlung von Lungenkarzinompatienten im Stadium IV ist zentrumsabhängig. Neben Zentren, die überwiegend mit Chemotherapie arbeiten, existieren auch Zentren mit einer hohen Rate an Supportivbehandlungen. Diese Supportivbehandlungen entsprechen sicherlich nicht dem heute in Leitlinien geforderten Standard, ihr vergleichsweise häufiger Einsatz lässt sich jedoch mit dem Studienbeginn bereits im Jahre 1997 erklären. Zudem erfolgt die Zuweisung von Patienten an einzelne Zentren zum Teil sehr gezielt je nach Spezialisierung der Kliniken, z. B. bei der Palliativbehandlung.

Die Ergebnisqualität in der HALLUCA-Studie ist in Abbildung 2 und Tabelle 4 dargestellt. Auffällig sind die im Vergleich zur Literatur deutlich schlechteren 3-Jahres-Überlebensraten in frühen
Stadien, die zum Teil mit der niedrigen Operationsrate zu erklären sind [2]. Der Vergleich der epidemiologisch gewonnenen HALLUCA-Daten mit der von Mountain [2] durchgeführten Analyse ist allerdings nur bedingt statthaft, da in diese Auswertung überwiegend in chirurgischen Kliniken diagnostizierte Stadien eingingen. Grundsätzlich muss daher die Erfassungsmethodik bei derartigen Vergleichen immer mit berücksichtigt werden.

\section{Qualitätssicherung in der Thoraxchirurgie}

Mit der Gründung der Kommission «Qualitätssicherung» im Jahr 1989 begann die Deutsche Gesellschaft für Thoraxchirurgie (DTG) bereits früh, sich dieses wichtigen Themas anzunehmen. In ersten ab 1990 initiierten Pilotstudien wurde zunächst in einzelnen Zentren die Häufigkeit chirurgischer Eingriffe in den verschiedenen Stadien des Lungenkarzinoms mit Komplikations- und Letalitätsraten erfasst [3]. Dabei stand in dieser frühen Phase die quantitative Datenerfassung im Vordergrund. Lassen sich die Kriterien für Prozessqualität (z. B. Zahl der Mediastinoskopien relativ zu Lobektomien und Pneumonektomien) und Ergebnisqualität (z. B. Letalitätsrate) noch relativ einfach definieren, so kann ein Qualitätsanforderungsprofil erst nach und nach im Rahmen von Maßnahmen zur Qualitätssicherung erarbeitet werden, da verbindliche Standards und Toleranzbreiten bislang nicht existieren.

In einer dritten, im Jahr 2000 durchgeführten größeren Pilotstudie wurden innerhalb von 3 Monaten an 7 Kliniken unterschiedlicher Spezialisierung insgesamt 413 Lungenkarzinompatienten erfasst. Auf Basis dieser Daten wurden anschließend Klinikprofile erstellt, die einen Vergleich der teilnehmenden Zentren hinsichtlich Art der durchgeführten Eingriffe und der Komplikationsraten erlauben. Diese Studie wies auf erhebliche Unterschiede zwischen den einzelnen Zentren hin.

Dieses Bild wurde durch eine größere, über 12 Monate laufende Studie mit 1099 an 6 Kliniken operierten Patienten bestätigt. Die Komplikationsraten bei den 1499 erfassten Operationen variierten von Klinik zu Klinik, bei allerdings weiten und überlappenden Konfidenzintervallen (Abb. 3a). Auch das Verhältnis von großen Eingriffen zu Mediastinoskopien zeigt mit Raten von $20-80 \%$ eine große Schwankungsbreite. Die Letalitätsrate bewegte sich im Gesamtkollektiv zwischen 1,5 und 3,5\% (Abb. 3b). Nicht überraschend war diese Quote bei den Pneumonektomien mit 8,1\% (4-33\%) höher als im Falle der Lobektomien mit rund 3\% (0-6,9\%). Die Zahlen sind vergleichbar mit Daten aus den USA und mit einer Statistik zur Operationsletalität in Nordrhein-Westfalen $[4,5]$.

\section{Qualitätssicherung in Baden-Württemberg initiiert}

Die im Rahmen der DTG erstellten Pilotstudien haben die Machbarkeit der Qualitätssicherung in der Thoraxchirurgie belegt. Ein erster Schritt auf diesem Weg wurde durch die Landesärztekammer Baden-Württemberg getan, die die Qualitätssicherung bei der 


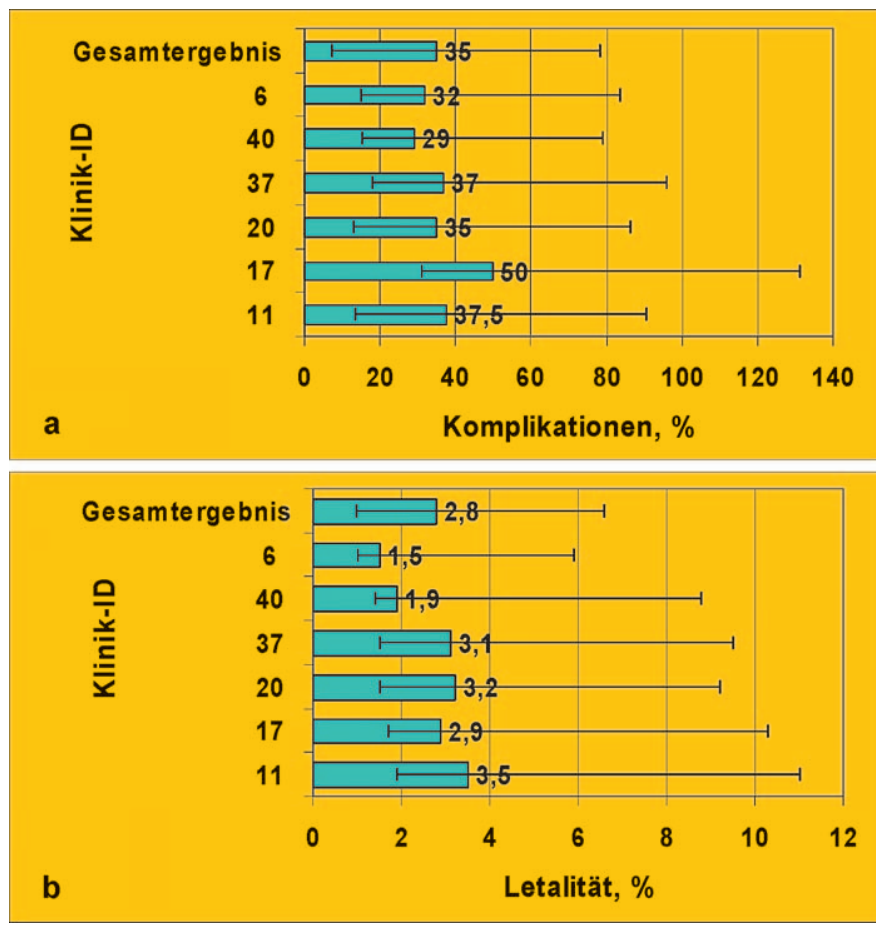

Abb. 3. Pneumonektomie $(n=148)$ in thoraxchirurgischen Abteilungen. a Komplikationsraten (14 Arrythmien, 9 Blutungen, 3 Stumpfinsuffizienzen), b Letalitätsraten (Friedel).

operativen Behandlung des Lungenkarzinoms genehmigt hat und über einen Zeitraum von 5 Jahren finanzieren will. Damit sind die Thoraxchirurgen die erste Gruppe, die die onkologische Ergebnisqualität systematisch anhand der 5-Jahres-Überlebensrate erfasst.

\section{Hohe Qualitätsstandards in der Strahlentherapie}

In der Strahlentherapie hat die Qualitätssicherung bereits ein hohes Niveau erreicht. Denn häufige und regelmäßige Checks von Dosimetrie, Isozentrums- und Blendengenauigkeit sowie Protokolle zur Regelüberprüfung von Laser-Positionierung, Simulator und Planungssystem sind seit geraumer Zeit etabliert. Jede Behandlung am Patienten wird anhand zahlreicher Parameter elektronisch verifiziert. Die einzelnen Behandlungsschritte und eventuelle Änderungen werden bildlich dokumentiert. Zusätzlich findet jährlich eine externe Überprüfung der Gerätetechnik durch den TÜV und von Dosimeter und Dosimetrie durch die Physikalisch-Technische Bundesanstalt Braunschweig statt. So ist sichergestellt, dass die Strahlendosis an einem definierten Punkt im Zielvolumen um maximal 3\% von der geplanten Dosis abweicht und sich die Genauigkeit der Feldeinstellung in einem Bereich von 5-7 mm - bei Präzisionstechniken wie der stereotaktischen Bestrahlung sogar noch deutlich darunter - bewegt.

Neben der physikalisch-technischen Qualitätssicherung wird jede strahlentherapeutische Abteilung im Rahmen zweijährlich stattfindender externer Audits auch auf ihre Behandlungsqualität hin analysiert. Hier wird an zufällig ausgewählten Patienten die gesamte

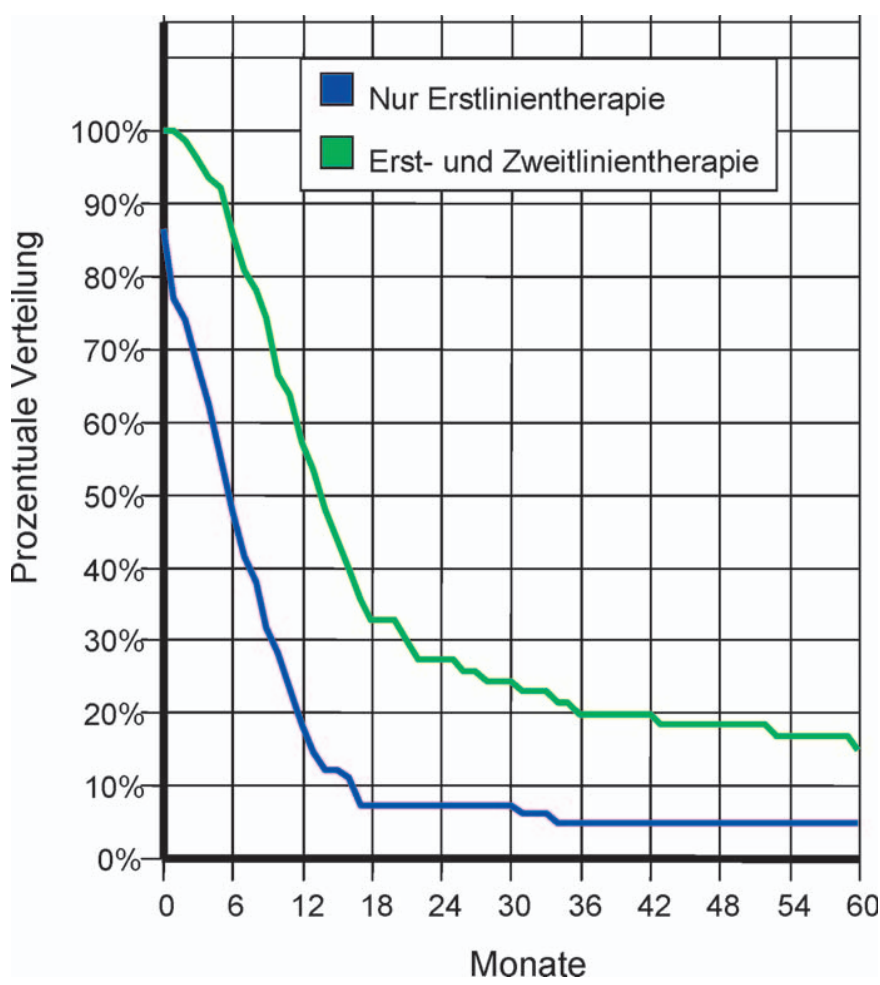

Abb. 4. Tumorregister Donaustauf: 5-Jahres-Überlebensdaten bei NSCLC-Patienten im Stadium IIIB/IV in Abhängigkeit von der Therapie (alleinige Erstlinientherapie ( $\mathrm{n}=97)$ vs. Erst- und Zweitlinientherapie $(\mathrm{n}=78)$ ) (Huber).

Behandlungskette innerhalb der Abteilung überprüft. Bei den 2005 an 35 strahlentherapeutischen Abteilungen in Bayern durchgeführten Audits schnitt die Physik sehr gut ab. Dagegen wurde im Bereich der Medizin teilweise Verbesserungsbedarf konstatiert.

\section{Aufgaben für die Zukunft}

Die Ergebnisqualität der Strahlentherapie wird innerhalb Bayerns flächendeckend im Rahmen von Qualitätszirkeln, z. B. anhand von lokaler Kontrolle oder Spätkomplikationen, erfasst. Diese Maßnahme ist für die Strahlentherapie des kolorektalen Karzinoms mittlerweile abgeschlossen; derzeit erhebt und analysiert man die Daten für das Prostatakarzinom.

Im Falle des Lungenkarzinoms sind allerdings noch einige grundsätzliche Fragen offen: So stellt sich das innerhalb von Studien vermutlich kaum zu lösende Problem, dass die Zielvolumina für eine Radiatio nach Positronenemissionstomogramm(PET)-Staging nicht genau definiert sind. Hier ist zu hoffen, dass die im Rahmen der Qualitätssicherung gewonnenen Daten zukünftig Vorgaben für ein optimiertes Vorgehen liefern werden. Auch die Etablierung eines exakten Algorithmus zur Standardisierung der für die Lunge schwierigen Dosisberechnung kann möglicherweise durch die Qualitätssicherung entscheidend vorangetrieben werden.

Grundsätzlich müssen die erhobenen Daten in einem klinischen Register gesammelt werden, zu dem sämtliche Beteiligten Zugriff haben - eine Forderung, die wahrscheinlich nur in Form Internet- 
basierter Register zu realisieren ist. Ebenfalls wichtig ist die langfristige Nachbeobachtung der erfassten Patienten, um so sicherstellen zu können, dass erarbeitete qualitätssichernde Maßnahmen auch in die Praxis implementiert werden.

\section{Fragebogen soll Status quo feststellen}

Die Qualitätsüberprüfung medizinischer Leistungen ist wichtig, muss sich aber letztlich in einer Prognoseverbesserung der Patienten aufgrund verbesserter diagnostischer und therapeutischer Maßnahmen niederschlagen. Die Daten des Tumorregisters Donaustauf für den Zeitraum von 1980-2003 belegen eine relativ stadienunabhängige Steigerung der 5-Jahres-Überlebensraten beim Lungenkarzinom um rund $10 \%$ - ein relevantes Ergebnis, das durch die Zahlen weiterer Tumorregister gesichert wird. Den Erfolg neuer therapeutischer Maßnahmen verdeutlichen die unterschiedlichen Überlebensraten von Lungenkarzinompatienten im Stadium IIIB/IV, die einer Zweitlinienchemotherapie zugeführt werden bzw. keine Rezidivtherapie erhalten (Abb. 4).

Allerdings war der Effekt neuer Therapien bislang begrenzt, so dass an weiteren Stellschrauben gedreht werden muss. Als wichtige veränderbare Einflussgröße hat sich die Therapiequalität herausgestellt. Um zunächst den Status quo bei der Versorgung von Lungenkarzinompatienten zu ermitteln, wurde eine Umfrage konzipiert, die das Management von Patienten mit Lungenkarzinom in Deutschland erfassen soll. Unter den Bedingungen z.B. des Bayerischen Krebsregistergesetzes wäre später auch eine anonymisierte patientenbezogene Auswertung vorstellbar.

\section{Literatur}

1 Roberts JR, et al: Prospective comparison of thoracoscopic and pathologic staging in patients with early stage non-small cell lung cancer. Ann Thorac Surg 1999;68:1154-1158.

2 Mountain CF: Revisions in the International System for Staging Lung Cancer. Chest 1997;111: 1710-1717.
3 Friedel G, Toomes H: Qualitätssicherung - Thoraxchirurgie. Acta Chir Austriaca 1996;28:116120.

4 Friedel G, et al: Qualitätssicherung in der Thoraxchirurgie anhand von Eingriffen beim Bronchialkarzinom. GMS Thor Surg Sci 2004;1: Doc02.
5 Bruckenberger E: Analyse der Vorgaben von Mindestmengen planbarer Leistungen nach dem Fallpauschalengesetz. Krankenhaus-Umschau, 25. 03. 2003.

www. bruckenberger.de/frame.htm?=literat.htm. 